\title{
Reconstruction of Stellar Orbits Close to Sagittarius A*: Possibilities for Testing General Relativity
}

\author{
P. Chris Fragile and Grant J. Mathews \\ University of Notre Dame, Center for Astrophysics, Notre Dame, IN 46556 \\ pfragile@nd.edu
}

\begin{abstract}
We have reconstructed possible orbits for a collection of stars located within 0.5 arcsec of Sgr A*. These orbits are constrained by observed stellar positions and angular proper motions. The construction of such orbits serves as a baseline from which to search for possible deviations due to the unseen mass distribution in the central 1000 AU of the Galaxy. We also discuss the likelihood that some of these stars may eventually exhibit detectable relativistic effects, allowing for interesting tests of general relativity around the $2.6 \times 10^{6} M_{\odot}$ central object.
\end{abstract}

Subject headings: black hole physics - celestial mechanics, stellar dynamics - Galaxy: center - Galaxy: kinematics and dynamics - gravitation — relativity

\section{Introduction}

The generally accepted idea that supermassive accreting black holes power the highly energetic phenomena in active galactic nuclei has motivated a great deal of effort to gather information about these extraordinary objects. Speculation about the presence of a black hole at the center of our own Galaxy has been ongoing for over 20 years [see Genzel et al. (1996) or Kormendy \& Richstone (1995) and references therein for recent summaries]. Although the Galactic center appears to be nearly radio dormant, there is ample evidence for the presence of a supermassive black hole. [See, however, Tsiklauri \& Viollier (1998) and Munyaneza, Tsiklauri, \& Viollier (1998) for discussions of other possible interpretations.]

One of the most efficient ways to constrain the possible existence of a massive object in the Galactic core is to carefully observe the orbital motions of the stars and gas closest to the Galactic center. Furthermore, deviations from ideal orbits may provide a probe of the distribution of dark matter around the Galactic center. Progress toward the accumulation of the relevant observational data has recently been made by Eckart et al. (1995), Genzel et al. (1996), Eckart \& Genzel (1997), Genzel et al. (1997), and Ghez et al. (1998) who have obtained high angular resolution K-band images of the Galaxy's central stellar cluster. 
Here we report on an attempt to reconstruct ideal orbits for the innermost 8 stars in the Ghez et al. (1998) survey using relativistic equations of motion. There is an obvious difficulty in determining orbital parameters for stars with periods much longer than the observational baseline, particularly when the uncertainties in the locations of the stars at each epoch are comparable to the apparent motion of the stars between observing epochs. Nonetheless, orbit reconstruction is possible in principle, since orbital mechanics is deterministic once the position and velocity vectors for the relevant masses are given at one instant of time.

The 8 stars studied here are the ones for which the possible general relativistic effects should be greatest. The high velocities of these stars, and as we shall see, the possibility of significant periapse precession could provide a groundwork for interesting tests of general relativity as future observations of these stars are made. We also discuss other possible detectable relativistic effects which could occur if the stars pass near the massive object. Jaroszyński (1998) has presented Monte Carlo simulations to demonstrate the feasibility of measuring such effects. Jaroszyński (1999) and Salim \& Gould (1999) also demonstrate how accurate determinations of the orbit parameters can be used to constrain distance and mass estimates for the Galactic center. Here, we assess what can and has been learned from the currently available data. This work can also serve as a foundation for future, more-detailed probes of the mass distribution of the Galactic core. It is important to initially compute the two-body orbits of these stars around the central massive object, without considering stellar interactions or mass distribution effects. These two-body orbits provide a welldefined dynamical model against which to compare the actual astrometry and velocities of the objects. Any discrepancy or lack thereof bears directly on the mass distribution around the central black hole and could serve as an indirect means to detect the dark matter distribution in the Galactic center. For completeness, we also consider the case in which a few percent of the mass is distributed on scales comparable to the semi-major axes of the orbits being considered. As will be seen, there are distinctly different observational consequences in each case.

In this paper, we present a summary of the available published astrometric data in $\S 2$. Section 3 describes our orbital solution technique, and $\S 4$ gives the constraints on the orbit parameters. In $\S 5$ we discuss the possible relativistic and hydrodynamic effects these orbits may display. In $\S$ 6 we discuss which observations would be most beneficial in future studies.

\section{Astrometric Data}

Genzel et al. (1997) (hereafter referred to as GEOE) have presented astrometric $K$-band maps of the central $3 \times 3 \operatorname{arcsec}^{2}$ of the Galaxy's central star cluster for five epochs between 1992 and 1996. These images were taken using the 3.5-m New Technology Telescope of the European Southern Observatory. Ghez et al. (1998) (hereafter referred to as GKMB) have presented $K$-band maps of the central $1 \times 1 \operatorname{arcsec}^{2}$ for three epochs between 1995 and 1997. Their data was taken using the W. M. Keck 10-m telescope. Each group reported on the RA and DEC separation from Sgr A* as well as the angular proper motions for the stars. Genzel et al. (2000) presents an updated 
tabular summary of the proper motions obtained to date. Fig. 1 shows the combined data for the 8 stars studied here. The error bars show the uncertainty in the centroid positions for each data point. The reported uncertainties were typically on the order of 0.008 arcsec for GEOE and 0.002 arcsec for GKMB. We followed the naming convention of GKMB, so the 8 stars studied here are given the labels S0-1 through S0-8. We have ignored the first epoch of data from GEOE (1992.65) because of the difficulty they had in resolving any of the individual stars in the central-most region during that observation. Also, star S0-8 was not evident in the 1994.27 epoch of the GEOE data. Both groups used the results from Menten et al. (1997) to determine the position of Sgr A*. This means that the RA and DEC positions from both studies are subject to the inherent uncertainty of that work. Although Menten et al. (1997) give an error estimate of 0.03 arcsec, the GKMB group was able to link the infrared and radio reference frames and use $2 \mathrm{SiO}$ maser sources to identify the position of Sgr $\mathrm{A}^{*}$ to within 0.01 arcsec.

\section{3. $\quad$ Fitting Method}

Because these stars have high velocities and may pass quite close to the supermassive object at the Galactic center, we choose to evolve the orbits using relativistic equations of motion for a test object of negligible mass orbiting a Schwarzschild or maximal Kerr black hole. Given the extremely large mass of the central object $\left(2.6 \pm 0.2 \times 10^{6} M_{\odot}\right)$, the approximation that the stars move in a fixed background metric is certainly acceptable. Jaroszyński (1998) has studied possible orbits of these stars in a Kerr background and concluded that the effects of black hole angular momentum are probably negligible. We also have made a study of orbits around a maximal Kerr black hole. As we will describe below, we found no detectable difference between the Kerr and Schwarzschild dynamics. On the other hand, Munyaneza et al. (1998) have demonstrated that noticeable differences in orbit characteristics are possible if an extended mass distribution is present instead of a single compact object. However, GKMB have placed significant constraints on the possibilities of an extended mass distribution. For this reason, we consider two separate cases: one in which all of the mass is contained in the central black hole and one in which roughly $5 \%$ of the central mass is contained in an ideal $(\gamma=5 / 3)$ gas cloud in hydrostatic equilibrium, distributed on a scale equal to the best-fit semi-major axis of star S0-2. The remaining $95 \%$ of the mass is still in a central black hole. The gravitational potentials for these two cases are plotted in Fig. 2. As more and better data become available, refinements to the mass distribution may be detectable. For illustration, the equations of motion in Schwarzschild coordinates with the origin fixed at Sgr A* are written (Weinberg 1972, Sec. $8.4 \&$ 11.1)

$$
\begin{aligned}
\frac{d^{2} r}{d \tau^{2}} & =\frac{-1}{2 A(r)} \frac{d A(r)}{d r}\left(\frac{d r}{d \tau}\right)^{2}+\frac{r}{A(r)}\left(\frac{d \theta}{d \tau}\right)^{2} \\
& +\frac{r \sin ^{2} \theta}{A(r)}\left(\frac{d \phi}{d \tau}\right)^{2}-\frac{1}{2 A(r)}\left(\frac{d B(r)}{d r}\right)\left(\frac{d t}{d \tau}\right)^{2}
\end{aligned}
$$




$$
\begin{gathered}
\frac{d^{2} \theta}{d \tau^{2}}=-\frac{2}{r} \frac{d \theta}{d \tau} \frac{d r}{d \tau}+\sin \theta \cos \theta\left(\frac{d \phi}{d \tau}\right)^{2}, \\
\frac{d^{2} \phi}{d \tau^{2}}=-\frac{2}{r} \frac{d \phi}{d \tau} \frac{d r}{d \tau}-2 \cot \theta\left(\frac{d \phi}{d \tau}\right)\left(\frac{d \theta}{d \tau}\right),
\end{gathered}
$$

and

$$
\frac{d^{2} t}{d \tau^{2}}=-\frac{d \ln B(r)}{d r}\left(\frac{d t}{d \tau}\right)\left(\frac{d r}{d \tau}\right)
$$

where the Schwarzschild metric parameters inside the mass distribution are

$$
A(r)=\left[1-\frac{2 \mathcal{M}(r)}{r}\right]^{-1}
$$

and

$$
B(r)=\exp \left\{-\int_{r}^{\infty} \frac{2}{r^{\prime 2}}\left[\mathcal{M}\left(r^{\prime}\right)+4 \pi r^{3} P\left(r^{\prime}\right)\right]\left[1-\frac{2 \mathcal{M}\left(r^{\prime}\right)}{r^{\prime}}\right]^{-1} d r^{\prime}\right\}
$$

where

$$
\mathcal{M}(r) \equiv \int_{0}^{r} 4 \pi r^{\prime 2} \rho\left(r^{\prime}\right) d r^{\prime}
$$

Outside the mass distribution,

$$
B(r)=A^{-1}(r)=1-\frac{2 \mathcal{M}(R)}{r} .
$$

Here $r, \theta$, and $\phi$ have their usual meanings, $\tau$ is the proper time, $t$ is the coordinate time, $\mathcal{M}(R)=M$ is the mass of the central object, $P$ is the proper pressure, and $\rho$ is the proper total energy density. Here and throughout we use the convention of Weinberg (1972), i.e. a time-like metric, a negative Riemann tensor, and a negative sign in the Einstein equation [cf. Misner, Thorne, \& Wheeler (1973), to convert to other conventions]. Also, we use geometrized units $(G=c=1)$ except where otherwise noted.

Setting up the problem thus means that the parameters which specify a model orbit are $\left\{r_{0}, \theta_{0}, \phi_{0}, \dot{r}_{0}, \dot{\theta}_{0}, \dot{\phi}_{0}\right\}$, i.e. the position and velocity components at some instant in time, $t_{0}$. We find these parameters using a least square minimization of the expression:

$$
\chi^{2}=\sum_{i=1}^{N} \frac{\left(\mathrm{RA}\left(t_{i} ; r_{0}, \theta_{0}, \phi_{0}, \dot{r}_{0}, \dot{\theta}_{0}, \dot{\phi}_{0}\right)-\mathrm{RA}_{i}\right)^{2}+\left(\mathrm{DEC}\left(t_{i} ; r_{0}, \theta_{0}, \phi_{0}, \dot{r}_{0}, \dot{\theta}_{0}, \dot{\phi}_{0}\right)-\mathrm{DEC}_{i}\right)^{2}}{\sigma_{i}^{2}}
$$

where $\mathrm{RA}_{i}$ and $\mathrm{DEC}_{i}$ are the measured RA and DEC offsets at epoch $t_{i}$ and RA $\left(t_{i} ; r_{0}, \theta_{0}, \phi_{0}, \dot{r}_{0}, \dot{\theta}_{0}, \dot{\phi}_{0}\right)$ and $\operatorname{DEC}\left(t_{i} ; r_{0}, \theta_{0}, \phi_{0}, \dot{r}_{0}, \dot{\theta}_{0}, \dot{\phi}_{0}\right)$ are the corresponding coordinate offsets for that same epoch, calculated using the model parameters. The error bars in the measured RA and DEC offsets are generally unequal. Therefore the best weighting factor for the goodness of fit is the radius of the 
error ellipse along the line formed by joining the observed position at an epoch with the modeled location of the star at that epoch. Hence, we write the weighting factor for the orbit fits as

$$
\sigma_{i}^{2}=\left(\sigma_{R A_{i}} \cos \Phi_{i}\right)^{2}+\left(\sigma_{D E C_{i}} \sin \Phi_{i}\right)^{2}
$$

where $\Phi_{i}$ is the angle between the RA-axis and the line drawn between the observed and modelorbit positions. To convert from angular separations to physical distances, we adopt $8.0( \pm 0.5) \mathrm{kpc}$ as the distance to the Galactic center (Reid, 1993). The uncertainty in this distance is included in our error estimates.

It is important to note that the orbits thus determined are degenerate in the parameters $\theta_{0}$ and $\dot{\theta}_{0}$. There is no way to determine from the available data whether or not a particular star is currently in front of or behind Sgr A*. By choice, we have assumed that all of these stars currently lie in front. This choice affects the orbit parameters given in the next section, but has no effect on the conclusions of this paper. Refined spectroscopic studies will help to break this degeneracy.

\section{Computed Orbits}

Having determined the model orbits as described above, we then convert the orbit parameters to their more familiar form $\{a, e, i, \Omega, \omega, T\}$, where $a$ is the semi-major axis, $e$ is the eccentricity, $i$ is the inclination, $\Omega$ is the longitude of the ascending node, $\omega$ is the argument of periapse, and $T$ is the time of last periapse passage [cf. Taff (1985), for the analytic expressions needed to perform this conversion]. Table 1 gives the orbit parameters for stars S0-1 through S0-3. Stars S0-4 through S0-8, though analyzed, are not listed in Table 1 because the orbit parameter $\theta_{0}$ for these stars is not constrained by the observations, i.e. all orbits with $0 \leq \theta_{0}<\pi$ are within $1 \sigma$ of the optimum

fit. For the convenience of the readers, we have included the period, $P$, for each of the orbits in Table 1. The errors given in the table are the statistical $1 \sigma$ uncertainties for each parameter. Star S0-1 is an interesting case because its "best-fit" orbit is actually an unbound, hyperbolic trajectory. Fig. 2 shows the computed, optimum least $\chi^{2}$ orbits overlaid on the observed data.

\section{Relativistic and Hydrodynamic effects}

The most relevant relativistic effect in a proper motion study is the precession of periapse. The angular advance of the periapse, evaluated numerically here, is given approximately by

$$
|\Delta \omega|=6 \pi \frac{G M}{a\left(1-e^{2}\right) c^{2}}
$$

in units of radians per revolution [cf. Ohanian \& Ruffini (1994)]. It is a simple matter to transform the angular advance of periapse in the plane of the orbit into the more easily measured angular shift of apoapse in the plane of the sky. Table 2 gives the periapse precession and the angular shift 
of apoapse for stars S0-2 and S0-3, assuming no mass distribution. For both stars, values near the $1 \sigma$ upper limits are in principle measurable with the current observational position accuracies. Fig. 4 illustrates possible precession effects over multiple revolutions of a model orbit for star S0-2. For this illustration, we show orbits confined to the plane of the sky $(i=0)$, although the effect looks similar for any inclination in the range, $-40^{\circ} \lesssim i \lesssim 40^{\circ}$. The $\chi^{2}$ for this orbit differs from the "best-fit" orbit by only 0.4. For comparison, we have shown a Newtonian orbit for two pointmasses (left panel), as well as relativistic orbits with and without distributed mass (right panel and middle panel, respectively). As expected, there is no precession in the Newtonian, point-mass limit. For the relativistic cases, there is a very important difference in the resulting precession. Relativistic periapse precession, by itself, causes an angular advance of periapse (middle panel). With the orbital inclination confined to small values, as in the model orbit illustrated here, the apoapse shift is 0.001 arcsec per revolution and the orbital period is 10 years. Using the current measured angular separation accuracy of \pm 0.002 arcsec, the apoapse shift in this case could in principle be detectable with a baseline of $\approx 20$ years of observation. The periapse precession due to a possible mass distribution, on the other hand, normally results in an angular regression (right panel). Therefore, a measured advance of periapse for these stars is most likely a relativistic effect. Such a measurement would also place strict limits on any mass distribution. Clearly this is a difficult measurement and one must carefully exclude competing effects from other masses around the central black hole. Nevertheless, it is worth searching for, since even determining the sign of the precession will reveal a great deal.

We also considered the effect of black hole angular momentum on this orbit. Any effect will be most pronounced when the angular momentum axis of the orbit is aligned (or anti-aligned) with the angular momentum axis of the black hole. In this case, both were chosen to lie along the line of sight. For the model orbit shown in Fig. 4, the effect of the black hole angular momentum amounts to a 0.0001 arcsec shift of the apoapse after 3 revolutions ( $\approx 30$ years). Thus it represents a $3 \%$ effect on top of the relativistic periapse precession. Since our $1 \sigma$ upper limit on the relativistic apoapse shift for S0-2 and S0-3 is 0.001 arcsec per revolution, the best-case $1 \sigma$ upper limit on the apoapse shift due to black hole angular momentum is 0.00003 arcsec per revolution. Measuring this effect would require observations on a time scale of hundreds of years at current astrometric accuracies. Hence, we conclude that the orbits of these stars can probably not be used to detect black hole angular momentum.

Another potentially measurable effect is the gravitational redshift $\left(\Delta \nu / \nu=-G M / c^{2} R\right)$ of the light emitted from these stars as they proceed through their orbits. Note, however, that the expected special relativistic red- and blueshifts for these stars can be much larger that any anticipated gravitational redshift and must therefore be precisely accounted for before any gravitational redshift will be discernible. Table 2 contains a summary of both of these effects.

If these stars are sufficiently massive, it is possible that gravitational radiation from their final inspiral and plunge may be detectable by space-based interferometry such as the proposed LISA mission (P. Bender 1999, private communication). Nevertheless, the time scale for angular 
momentum loss by gravitational radiation for the orbits considered here is greater than a Hubble time ( $\left.\sim 10^{15} \mathrm{yr}\right)$. The most likely cause of the final plunge will be scattering by a 2 -body interaction somewhere along the orbit.

This raises the issue as to whether the time scale for these stars to suffer small angle deflections from other stars around the black hole is less than or comparable to the time scale necessary to carry out a measurement of the periapse precession. The magnitude of the acceleration on star $i$ due to the other stars in the field

$$
a=\sum_{j} \frac{m_{j}}{r_{i j}^{2}}
$$

will be dominated at any time by the nearest stars. For the observed stars, the typical nearest neighbor is separated by about 0.2 arcsec on the sky or about $0.008 \mathrm{pc}$ at $8 \mathrm{kpc}$. Allowing for eight times as many other (non K-luminous) stars to be present, the typical separation might be $0.004 \mathrm{pc}$ with an associated mean acceleration due to nearest neighbors of $\langle a\rangle \sim 9 \times 10^{-9} \mathrm{~m} / \mathrm{sec}$. Assuming the most pessimistic case that there is always a $1 \mathrm{M}_{\odot}$ star at this closest distance with the same direction, then the time scale for a star to be deflected by a minimally detectable 0.002 $\operatorname{arcsec}(\approx 16 \mathrm{AU})$ shift is then

$$
t=\sqrt{2 \Delta x /\langle a\rangle} \approx 700 \mathrm{yrs}
$$

Also, it should be noted that due to the motion around the black hole, these stars are probably moving with rapid relative velocities $v \sim 0.01 c$. Therefore, the radius of gravitational influence $G M_{\odot} /\left\langle v^{2}\right\rangle$ is quite small $\left(\sim 10^{4} \mathrm{~km}\right)$. Hence, unless there are extremely close encounters, we do not expect the mutual interactions to dominate over the relativistic periapse precession of interest here. On the other hand, any such deviation, if detected, would most likely be distinguishable from periapse precession in both direction and magnitude and would serve as a means to detect the presence of unseen matter.

The possibility of gravitational lensing of stars near the the Galactic center has been discussed elsewhere (Alexander \& Sternberg 1998; Salim \& Gould 1999; Jaroszyński 1999; and Capozziello \& Iovane 1999). It has been concluded that this effect is probably negligible (Jaroszyński 1999).

As another point of possible interest we consider the hydrodynamic effects of these stars passing close to the central object. For a star of mass $M_{*} \ll M_{B H}$, the Roche-lobe radius, $R$, is given by

$$
\frac{R}{r}=0.49\left(\frac{M_{*}}{M_{B H}}\right)^{1 / 3},
$$

where $r$ is the radial distance from the star to the central object and $M_{B H}$ is the mass of the central object (King \& Done 1993). This effect is strongly dependent on the periapse separation of these stars. It is reassuring that none of the optimum fits in the present study are passing so close that they might have experienced Roche-lobe overflow. Also, since it is now evident (Figer et al. 2000; Eckart, Ott, \& Genzel 1999) that these stars are probably OB stars and not extended K-giants, Roche overflow seems unlikely. Nevertheless, within the $1 \sigma$ uncertainties deduced here, it is at least possible that one or more of these stars might approach or temporarily exceed their Roche limit 
during the next periapse passage. This might be interesting to watch for, and if it occurred, it might lead to a burst of activity in the Galactic center.

As another possible observable effect, we consider whether any of these stars could pass close enough to the central black hole to experience hydrodynamic distortion due to tidal interactions and whether these distortions could lead to observable oscillations in surface temperature or luminosity. For distortions from an equilibrium spherical shape of the form

$$
r=R+\epsilon Y_{l}^{m}(\phi, \theta)
$$

where $Y_{l}^{m}(\phi, \theta)$ are spherical harmonics, both the oscillatory period and the excitation time scale for these Kelvin modes (Lang 1999) should be of order

$$
P=\frac{2 \pi}{\left[2 l(l-1) G M_{*} /(2 l+1) R^{3}\right]^{1 / 2}} .
$$

We estimate $P \lesssim 3$ days, which is too short compared to the time of periapse passage for such oscillations to be excited.

\section{Discussion}

Given the interesting physics that might be gleaned from extended observations of these orbits, it is useful to summarize what steps must be taken to reduce the large statistical uncertainties in the orbital parameters. Of course, a great deal is to be learned by continued astrometry. This is ultimately the only way in which the periapse precessions and/or effects of the mass distribution can be determined. In addition, it is crucial to obtain accurate radial line-of sight velocities as well as the radial distance to Sgr $\mathrm{A}^{*}$. Current studies have provided fairly accurate measurements of RA, DEC, $V_{R A}$, and $V_{D E C}$, however, these must be complemented by K-band spectroscopy. Eckart et al. (1999) and Figer et al. (2000) have reported on high resolution infrared spectroscopy in the vicinity of Sgr $\mathrm{A}^{*}$. The spectra are consistent with all of the stars in this sample being OB stars. The strongest feature observed from this region is a weak $\operatorname{Br} \gamma$ emission line. However, as of yet no individual redshifts for these stars have been identified. What is needed are long integrations with high spatial resolution. Clearly, it should be a high priority to obtain such redshifts. If all six coordinates could be measured to comparable precision (e.g. $\sigma_{x}=\sigma_{y}=\sigma_{z}=0.002 \operatorname{arcsec} \approx 20 \mathrm{AU}$ and $\sigma_{V_{x}}=\sigma_{V_{y}}=\sigma_{V_{z}} \approx 100 \mathrm{~km} \mathrm{~s}^{-1}$ ) then the $1 \sigma$ uncertainties in the orbit parameters for star S0-2 would reduce to about $25 \%$ of the best-fit values derived in this work. If the velocity uncertainties were reduced to $50 \mathrm{~km} \mathrm{~s}^{-1}$ then the uncertainties in orbit parameters for S0-2 would be reduced to about $20 \%$.

If the line-of-sight velocity for each star could be accurately obtained directly from spectroscopic studies then the change in radial velocity, $d V_{z} / d t=-G M z /\left(x^{2}+y^{2}+z^{2}\right)^{3 / 2}$, over time might be used to find a value for the final unknown parameter - the line-of-sight separation between the star and Sgr A* (labeled $z$ ). Fig. 4 illustrates graphically the relation between $d V_{z} / d t$ and $z$ for 
each of the stars. From this we see that, in order to measure $z$ to an accuracy of $20 \mathrm{AU}, d V_{z} / d t$ must be measured to an accuracy of $\sim 7.5 \mathrm{~km} \mathrm{~s}^{-1} \mathrm{yr}^{-1}$, in the case of the star S0-2. This would require, for instance, two measurements of $V_{z}$ at accuracies of $50 \mathrm{~km} \mathrm{~s}^{-1}$ separated by about 9.5 years. This time scale for accurate orbit determination is similar to the time scale derived by Salim \& Gould (1999) to use these orbits to better constrain the distance to Sgr A*.

\section{Conclusion}

We have explored families of possible orbits for the 8 known stars located within 0.5 arcsec of the Galactic center. Because line-of-sight velocities have not yet been obtained and only positions through the 1998 observing epoch are available, orbital parameters could only be constrained for 3 of the stars. The ideal orbits of these stars could be much better constrained from relatively short baseline studies of line-of-sight velocities, although continued observations of angular positions and angular proper motions are crucial if relativistic or mass-distribution effects are to be identified. These orbits as they now stand have at least the potential to display some extraordinary properties, including periods of less than 10 years and very high eccentricities. The current range of orbit parameters also allow for the possibility of interesting close encounters with the $2.6 \times 10^{6} M_{\odot}$ object at the Galactic core.

It is clear from Eckart et al. (1999) and Figer et al. (2000) that these stars are probably blue, luminous, and very young OB stars most likely formed from material infalling toward the black hole. A likely explanation is tidal disruption of infalling rich OB associations by the central black hole. Hence, it is reasonable for the stars to have the highly eccentric orbits deduced here.

The possibility of these eccentric orbits passing close to the black hole opens up the possibility for detection of relativistic effects such as periapse precession and/or gravitational redshift. At the same time, continued observations may also reveal the presence and distribution of unseen matter at the Galactic core. Hydrodynamic effects may be evident as well, if the stars pass close enough to be tidally distorted. Also, the star currently passing closest to $\operatorname{Sgr} \mathrm{A}^{*}$ in the plane of the sky may not be in a bound orbit around the central mass. This introduces the intriguing questions as to whether other members of this association are unbound. If so, then the inferred virial mass of the central object may need revision.

In light of the many interesting puzzles highlighted in this paper, it is imperative that priority be given to continued astrometric, as well as spectroscopic, study of these stars. Such studies will provide valuable knowledge about the distribution and the dynamical evolution of mass within the central core of the Galaxy.

The authors wish to acknowledge useful discussion with A. Ghez. We would also like to thank an anonymous referee for numerous helpful comments, particularly in regards to the importance of mass distribution effects. This work was supported by the National Science Foundation under 
grant PHY-97-22086. 


\section{REFERENCES}

Alexander, T. \& Sternberg, A. 1999, ApJ, 520, 137

Blum, R. D., Sellgren, K., \& DePoy, D. L. 1996, ApJ, 470, 864

Capozziello, S. \& Iovane, G. 1999, Phys. Lett., A259, 185

Eckart, A. \& Genzel, R. 1997, MNRAS, 284, 576

Eckart, A., Genzel, R., Hofmann, R., Sams, J. S., \& Tacconi-Garman, L. E. 1995, ApJ, 445, L23

Eckart, A., Ott, T., \& Genzel, R. 1999, A\&A, 352, L22

Figer, D. F., et al. 2000, ApJ, 533, L49

Genzel, R., Eckart, A., Ott, T., \& Eisenhauer, F. 1997, MNRAS, 291, 219

Genzel, R., Pichon, C., Eckart, A., Gerhard, O. E., \& Ott, T. 2000, MNRAS, submitted

Genzel, R., Thatte, N., Krabbe, A., Kroker, H., \& Tacconi-Garman, L. E. 1996, ApJ, 472, 153

Ghez, A. M., Klein, B. L., Morris, M., \& Becklin, E. E. 1998, ApJ, 509, 678

Jaroszyński, M. 1998, Acta Astronomica, 48, 653

Jaroszyński, M. 1999, ApJ, 521, 591

King, A.R. \& Done, C. 1993, MNRAS, 264, 388

Kormendy, J. \& Richstone, D. 1995, ARA\&A, 33, 581

Lang, K. R. 1999, Astrophysical Formulae (New York: Springer)

Menten, K. M., Reid, M. J., Eckart, A., \& Genzel, R. 1997, ApJ, 475, L111

Misner, C. W., Thorne, K. S., \& Wheeler, J. A. 1973, Gravitation (New York: W. H. Freeman and Company)

Munyaneza, F. Tsiklauri, D., \& Viollier, R. D. 1998, ApJ, 509, L105

Ohanian, H. C. \& Ruffini, R. 1994, Gravitation and Spacetime (New York: W. W. Norton \& Company)

Reid, M. 1993, ARA\&A, 31, 345

Salim, S. \& Gould, A. 1999, ApJ, 523, 633

Taff, L. G. 1985, Celestial Mechanics: A Computational Guide for the Practitioner (New York: John Wiley \& Sons) 
Tsiklauri, D., \& Viollier, R. D. 1998, ApJ, 500, 591

Weinberg, S. 1972, Gravitation and Cosmology: Principles and Applications of the General Theory of Relativity (New York: John Wiley \& Sons)

This preprint was prepared with the AAS LATEX macros v5.0. 
Fig. 1. - The measured positions of stars S0-1 through S0-8 based on the data of Genzel et al. (1997) (1994.27, 1995.60, 1996.25, and 1996.43 epochs) and Ghez et al. (1998) (1995.44, 1996.49, and 1997.36 epochs).

Fig. 2.- Gravitational potential (in geometrized units) for the two mass distributions considered in this paper. The lower curve is the potential for a single $2.6 \times 10^{6} M_{\odot}$ black hole at the Galactic center. The upper curve is the potential for a $2.47 \times 10^{6} M_{\odot}$ black hole with $0.13 \times 10^{6} M_{\odot}$ of matter in a hydrostatic gas cloud extending out to $1000 \mathrm{AU}$.

Fig. 3.- Best-fit orbits for S0-1 through S0-3. For S0-1, the best fit is actually an unbound, hyperbolic trajectory.

Fig. 4.- Multi-revolution trace of a possible model orbit for star S0-2 confined to the plane of the sky $(i=0)$. In all 3 panels, the initial orbit parameters are the same and the star orbits in a clockwise direction. Left panel: Newtonian orbit for point particles (no precession). Middle panel: angular advance of periapse (apoapse shifts to the right) for ideal test particle orbit around Schwarzschild black hole (no mass distribution). Right panel: angular regression of periapse (apoapse shifts to the left) due to the distribution of roughly $5 \%$ of the central mass over a volume on the order of the size of the test orbit.

Fig. 5.- Instantaneous time derivative of the line-of-sight velocity as a function of the line-of-sight separation from the central massive object. 
Table 1. Best-fit Orbit Parameters

\begin{tabular}{cccccccc}
\hline \hline Star ID & $a\left(10^{3} \mathrm{AU}\right)$ & $P(\mathrm{yrs})$ & $e$ & $T($ year $)$ & $i(\operatorname{deg})$ & $\Omega(\operatorname{deg})$ & $\omega(\operatorname{deg})$ \\
\hline S0-1 & $\infty$ & $\infty$ & $1.8( \pm 3.7)$ & $\mathrm{NA}$ & $112( \pm 6)$ & $213( \pm 30)$ & $193( \pm 327)$ \\
S0-2 & $1\left(_{-6}^{+11}\right)$ & $19\left(_{-185}^{+312}\right)$ & $0.5\left(_{-5.0}^{+9.8}\right)$ & $1981( \pm 304)$ & $106( \pm 96)$ & $269( \pm 16)$ & $312\left({ }_{-1300}^{+2870}\right)$ \\
S0-3 & $3\left(_{-175}^{+183}\right)$ & $80\left(_{-8260}^{+8650}\right)$ & $0.5\left(_{-56}^{+59}\right)$ & $1988( \pm 1810)$ & $96( \pm 44)$ & $335( \pm 1220)$ & $89( \pm 3720)$ \\
\hline
\end{tabular}


Table 2. Predicted Relativistic Effects

\begin{tabular}{|c|c|c|c|c|c|c|c|c|}
\hline Star ID & $\begin{array}{l}\text { Periapse } \\
\left(10^{2} \mathrm{AU}\right)\end{array}$ & $\begin{array}{l}\text { Apoapse } \\
\left(10^{2} \mathrm{AU}\right)\end{array}$ & $\begin{array}{c}\text { Periapse } \\
\text { Precession }^{\mathrm{a}} \\
(\mathrm{deg} / \mathrm{rev})\end{array}$ & $\begin{array}{l}\text { Apoapse } \\
\text { Shift }^{\mathrm{b}} \\
\text { (mas/rev) }\end{array}$ & $\begin{array}{c}{[\Delta \nu / \nu]_{G R}} \\
\text { at Periapse } \\
\quad\left(\times 10^{-5}\right)\end{array}$ & $\begin{array}{c}{[\Delta \nu / \nu]_{G R}} \\
\text { at Apoapse } \\
\quad\left(\times 10^{-5}\right)\end{array}$ & $\begin{array}{c}\quad|\Delta \nu / \nu|_{S R} \\
\text { at Periapse }{ }^{\mathrm{d}} \\
\quad\left(\times 10^{-3}\right)\end{array}$ & $\begin{array}{c}|\Delta \nu / \nu|_{S R} \\
\text { at Apoapse } \\
\quad\left(\times 10^{-3}\right)\end{array}$ \\
\hline S0-2 & $5\left(\begin{array}{c}+301 \\
-179\end{array}\right)$ & $15\left(\begin{array}{l}+515 \\
-305\end{array}\right)$ & $0.04\left({ }_{-0.25}^{+0.42}\right)$ & $0.03\left({ }_{-0.74}^{+1.27}\right)$ & $-5\left(\begin{array}{c}+202 \\
-341\end{array}\right)$ & $-2\left(\begin{array}{c}+35 \\
-59\end{array}\right)$ & $6\left(\left(_{-24}^{+41}\right)\right.$ & $2( \pm 24)$ \\
\hline S0-3 & $13( \pm 455)$ & $38( \pm 3,210)$ & $0.01\left({ }_{-0.96}^{+1.01}\right)$ & $0.01\left(\begin{array}{l}+1.28 \\
-1.22\end{array}\right)$ & $-2\left(\begin{array}{l}+62 \\
-65\end{array}\right)$ & $-1\left(\begin{array}{l}+55 \\
-58\end{array}\right)$ & $0.1( \pm 79.9)$ & $0.02( \pm 80.1)$ \\
\hline
\end{tabular}

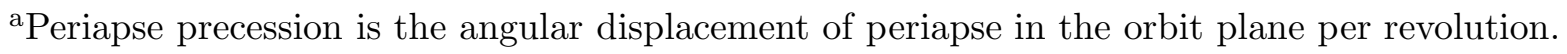

${ }^{b}$ Apoapse shift is the apparent motion of apoapse on the sky as seen by an observer on Earth.

${ }^{\mathrm{c}}$ Gravitational Redshift.

${ }^{\mathrm{d}}$ Relativistic Doppler Shift. The sign can not be determined from available data. 


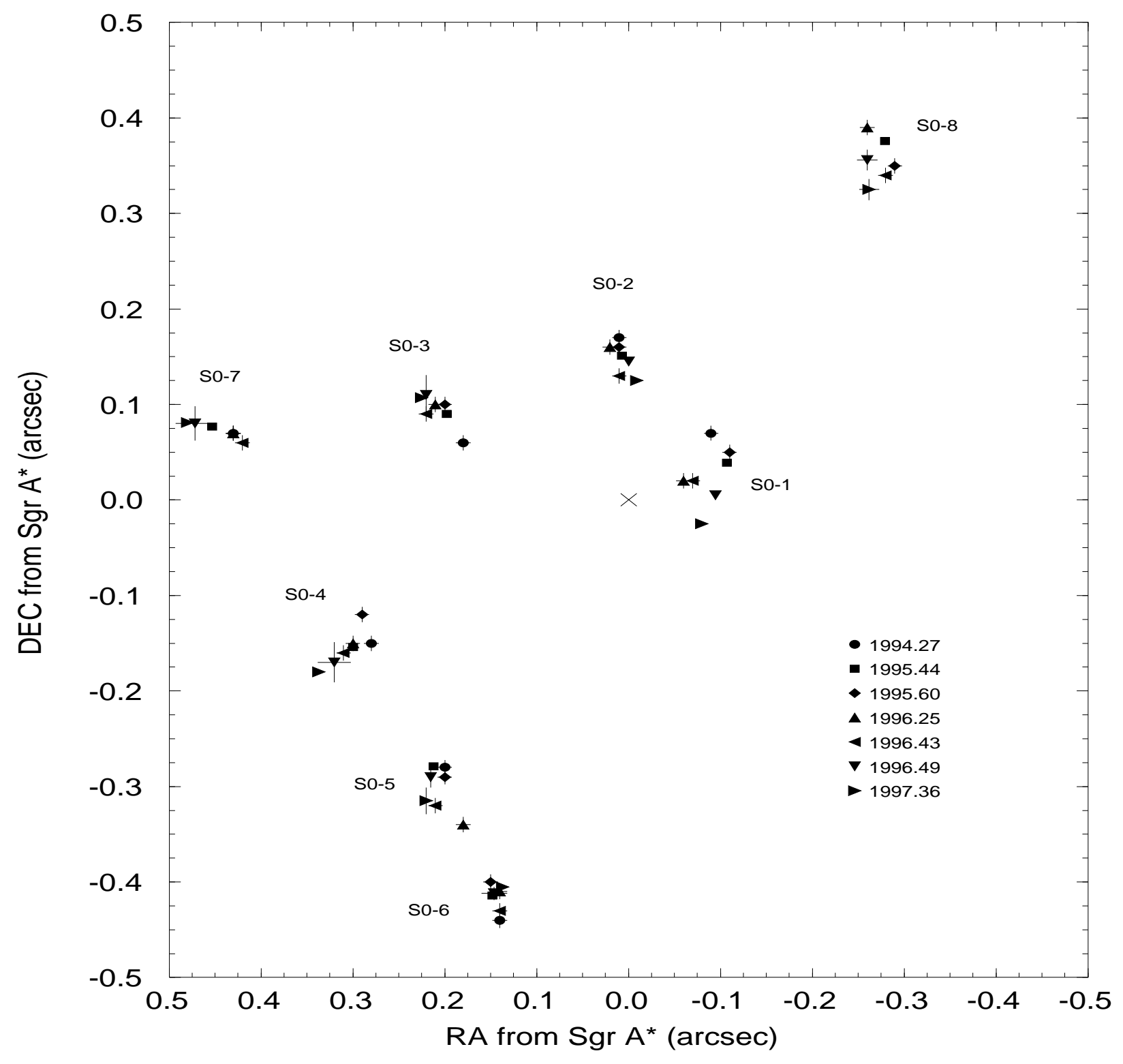




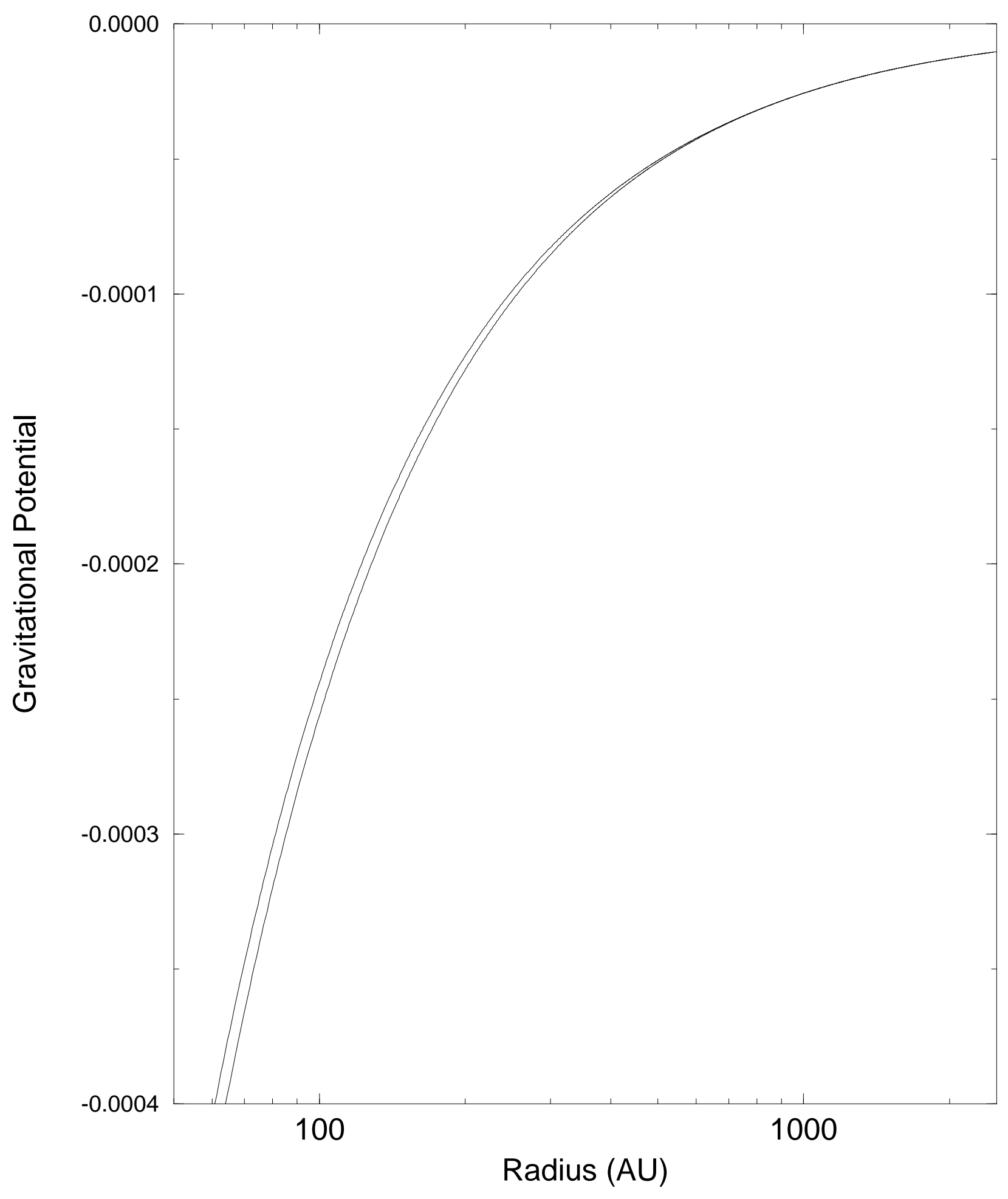




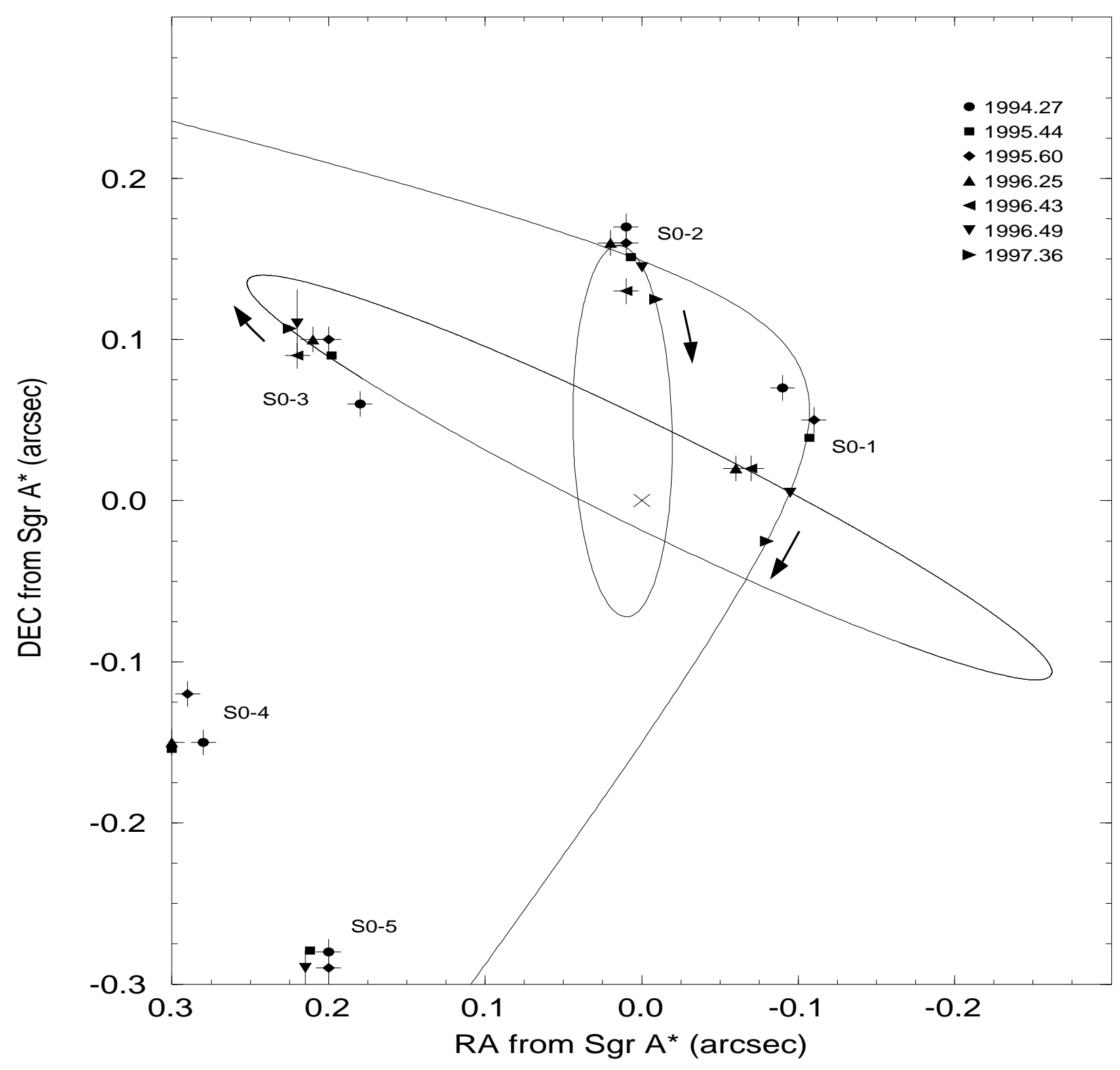




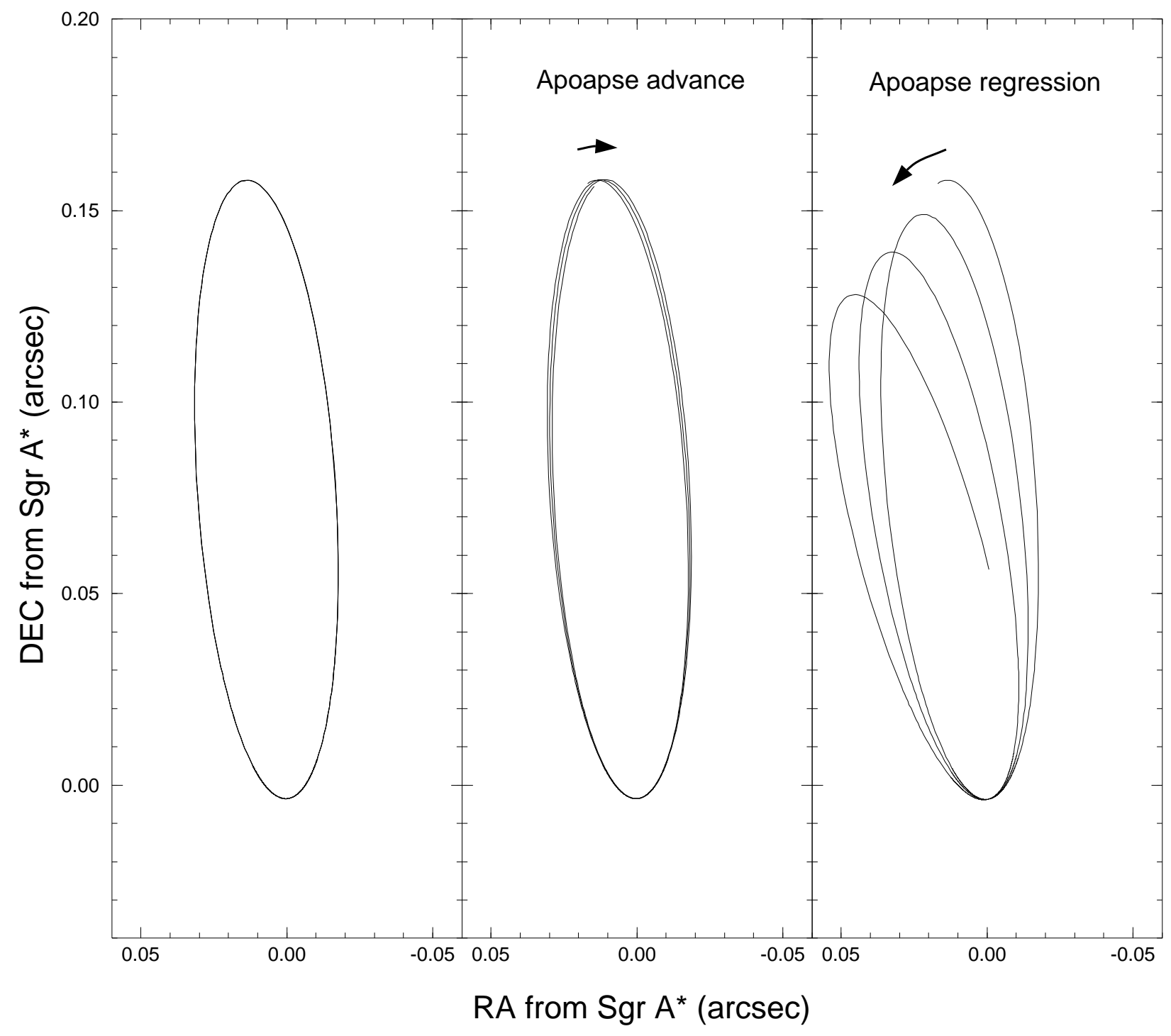




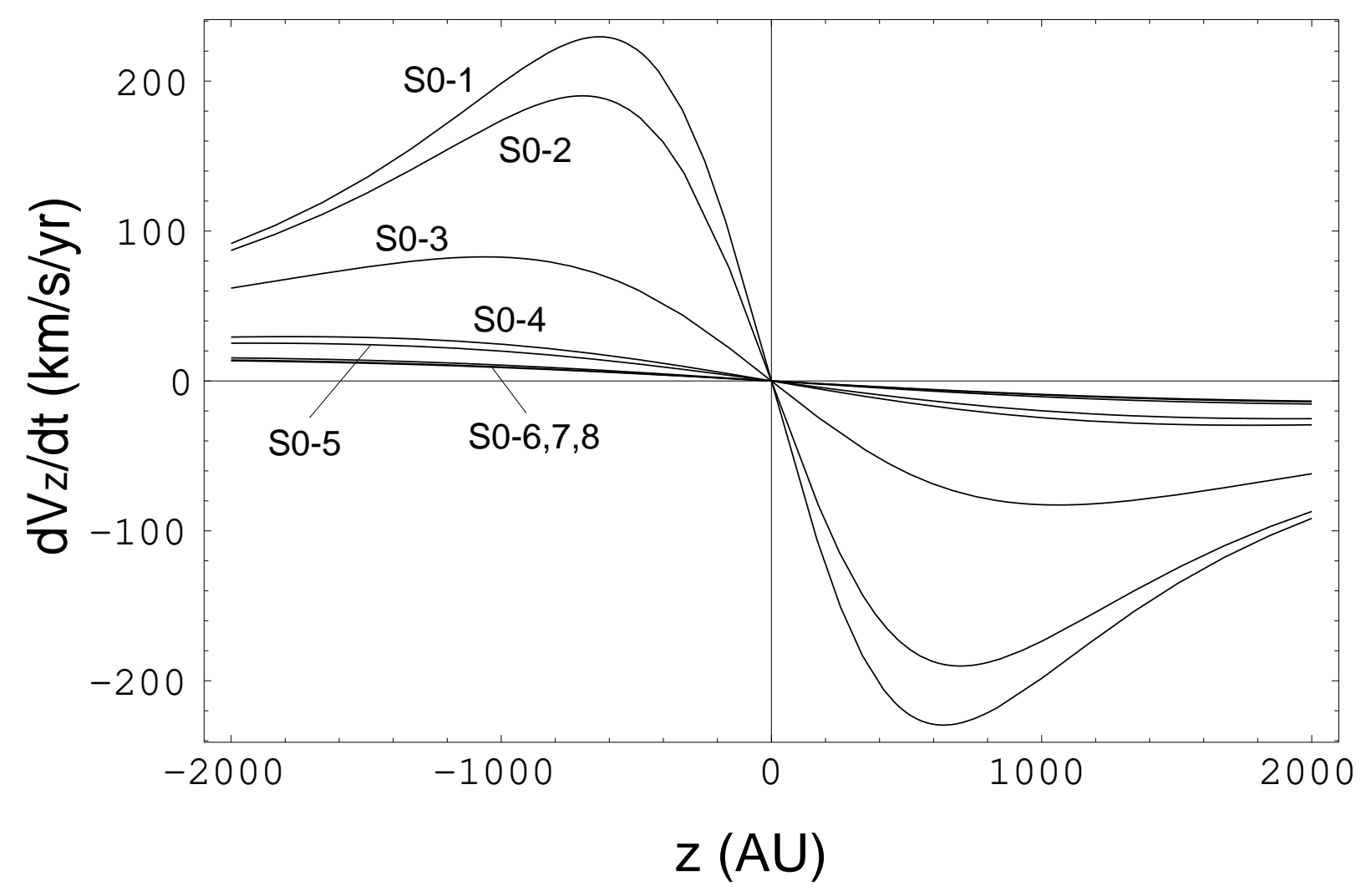

